\title{
Structural Analysis and Immunogenicity of Pseudomonas aeruginosa Immunotype 2 High Molecular Weight Polysaccharide
}

\author{
Gerald B. Pier and Susan E. Bennett \\ Channing Laboratory, Brigham and Women's Hospital, Department of Medicine, Harvard Medical School; Division of General Medicine, \\ Beth Israel Hospital; and the Harvard-Thorndike Laboratory, Boston, Massachusetts 02115
}

\begin{abstract}
We analyzed high molecular weight polysaccharide (PS) from the Fisher immunotype 2 (IT-2) strain of Pseudomonas aeruginosa for molecular composition and structure, then determined its immunogenicity in healthy adults. The PS was composed of 2-acetamido-2,6-dideoxygalactose ( $N$-acetyl fucosamine) and glucose in a molar ratio of 2:1. Structural analysis by carbon13 and proton nuclear magnetic resonance confirmed that the high molecular weight PS was structurally identical to that of the O-specific side chain of the lipopolysaccharide. PS differed from this material in molecular size. Immunization of 19 adult volunteers with doses of 50-100 $\mu \mathrm{g}$ of PS resulted in significant rises $(P<0.04-P<0.0001)$ in binding antibody levels and killing antibody titers 2 and 4 wk postimmunization. The only reaction to the vaccine was localized tenderness at the immunization site. Analysis of the immunoglobulin isotype response to the vaccine showed a rise in specific serum IgG and IgA antibodies. Heterologous responses to other $P$. aeruginosa $P S$ antigens were not seen. The antibody levels attained by vaccination were comparable with those in acute-phase serum samples of patients who survived sepsis with IT-2 $P$. aeruginosa and were significantly higher $(P<0.03)$ than specific antibody levels in bacteremic patients who died. These results confirm that PS is a high molecular weight, immunogenic form of the $P$. aeruginosa IT-2 serotype antigen, eliciting levels of type-specific antibody comparable with those seen among patients surviving an episode of $P$. aeruginosa sepsis.
\end{abstract}

\section{Introduction}

Infections caused by $P$. aeruginosa may occur during the course of cystic fibrosis and a variety of diseases characterized by a compromised immune system $(1,2)$. Not infrequently, $P$. aeruginosa infections constitute terminal events under these circumstances. Treatment of such infections is often hampered by the severity of the predisposing illness $(3,4)$ and by the resistance of most strains to multiple antibiotics $(5,6)$. Several lines of evidence indicate that immunity to $P$. aeruginosa is mediated principally by serotype-specific antibody. Serotype antigens are expressed on the O-specific side chain of the lipopolysaccharide

Address reprint requests to Dr. Pier, Channing Laboratory, 180 Longwood Ave., Boston, MA 02115. 1985.

Received for publication 26 June 1985 and in revised form 21 October

J. Clin. Invest.

(c) The American Society for Clinical Investigation, Inc.

0021-9738/86/02/0491/05, \$1.00

Volume 77, February 1986, 491-495
(LPS). ${ }^{1}$ Research to date revealed that humoral immune responses after infection with $P$. aeruginosa are directed at serotype antigens $(7,8)$. Animal studies using LPS vaccines have demonstrated a protective effect of type-specific antibody (9). There is an association between high levels of type-specific antibody in acute-phase serum and survival from $P$. aeruginosa sepsis $(10,11)$. Nevertheless, $P$. aeruginosa LPS vaccines suffer from inherent toxicity (12) and a lack of characterization of all of the vaccine components (13).

We have purified a high molecular weight polysaccharide (PS) component of $P$. aeruginos $a$ that appears to be a promising material for inducing serotype-specific immunity (14-16). High molecular weight PS is so named because it is serologically and chemically related to the LPS side chain, but it routinely has an average molecular weight of $>100,000$. PS lacks detectable lipid or LPS core sugars and is not toxic or pyrogenic in animals (14, 15). The safety and immunogenicity of three different preparations of high molecular weight PS from the immunotype 1 (IT-1) strain of $P$. aeruginosa have previously been demonstrated in humans $(17,18)$. The current study employing a single preparation of the immunotype 2 (IT-2) PS was undertaken for the following reasons: (a) to establish firmly the relationship of the IT-2 high molecular weight PS to the O-specific side chain; (b) to evaluate the safety and immunogenicity of IT-2 PS; and (c) to compare the serum concentration of antibody obtained by immunization with that observed in patients who survive an episode of sepsis with IT-2 P. aeruginosa.

\section{Methods}

Strains. P. aeruginosa strain $9882-80$, a human bacteremia isolate, was obtained from the Clinical Microbiology Laboratory of the Brigham and Women's Hospital, Boston, MA. This isolate was determined to be a Fisher IT-2 strain by agglutination (19). Strain $9882-80$ was used both as a source of PS and as the target bacterium in the opsonophagocytic assay.

Vaccine. High molecular weight PS antigen was prepared as previously described in detail (17). A mannan component was removed from the vaccine as described (18). This material was weighed, dissolved in pyrogenfree water with $1: 30,000$ Merthiolate at $0.2 \mathrm{mg} / \mathrm{ml}$, sterilized by passage through $0.45-\mu \mathrm{m}$ filters, and lyophilized in individual vials containing $0.2 \mathrm{mg} /$ vial. Sterility of the vaccine was ascertained in bulk before packaging and in $10 \%$ of the total number of vials, in accordance with Food and Drug Administration regulations (Title 21, Sect. 610.12). When the final product from three vials was assayed for recovery of the starting material by analyses of the glucose content (20), 99.2\% of the expected amount was present. Before use, vaccines were reconstituted with an appropriate amount of sterile saline for injection such that the desired dosage was contained in a volume of $0.5 \mathrm{ml}$.

1. Abbreviations used in this paper: IT-1, IT-2, and IT-7, immunotypes 1, 2, and 7; LPS, lipopolysaccharide; nmr, nuclear magnetic resonance; ppm, parts per million; PS, polysaccharide; RIP, radioimmunoprecipitin. 
Chemical analyses. Analyses for nucleic acids, protein, LPS, lipids, and monosaccharide constituents were performed on the vaccine before packaging for human use as described elsewhere (16). The $N$-acetyl fucosamine standard used in the gas-liquid chromatographic analysis was a generous gift of Dr. H. Jennings, National Research Council of Canada, Ottowa, Canada. Proton and carbon-13 nuclear magnetic resonance (nmr) studies were performed in the laboratory of Dr. Derrick Horton (Ohio State University, Columbus, $\mathrm{OH}$ ) by use of previously described methods (21). The average molecular weight of the PS vaccine before packaging for human use was measured by high performance liquid chromatography. Dextran standards of known average molecular weight (Pharmacia Fine Chemicals, Uppsala, Sweden) were applied to an I-125 sizing column (Waters Associates, Milford, MA) and the midpoint (peak) of the elution volume measured. This was plotted vs. the $\log _{10}$ of the average molecular weight of the standards and a linear regression formula calculated $\left(r^{2}=0.997\right)$. This formula was used to determine the average molecular weight of the IT-2 PS vaccine based upon the midpoint of its elution volume from the $1-125$ column.

Animal toxicity studies. The general safety tests in guinea pigs and mice were performed as prescribed by Food and Drug Administration regulations (Title 21, Sect. 610.11). Pyrogenicity in rabbits and endotoxin levels (measured by coagulation of a limulus lysate) were measured as previously described (17).

Volunteers. 20 healthy young adults were recruited to participate in the study through advertising on a bulletin board at a local college. Laboratory personnel were also allowed to participate. A physician obtained informed consent and a brief medical history from each subject. Subjects were assigned to one of three groups to be given different doses of vaccine. $20 \mathrm{ml}$ of blood were obtained by venipuncture before a $0.5-\mathrm{ml}$ subcutaneous injection given in the deltoid region of the arm. Subjects were interviewed 24 and $48 \mathrm{~h}$ after the injection to determine reactions, including fever. Postimmunization sera were obtained $\sim 2$ and 4 wk after injection.

Patient sera. Acute-phase sera from a variety of patients with IT-2 $P$. aeruginosa sepsis were obtained courtesy of Dr. Lowell Young, University of California, Los Angeles, CA; Dr. Matthew Pollock, Uniformed Services University of the Health Sciences, Bethesda, MD; Dr. George Jacoby, Massachusetts General Hospital, Boston, MA; and from patients at the Brigham and Women's Hospital, Boston, MA. Corresponding blood isolates were obtained from the clinical microbiology laboratories and serotyped by agglutination (19). Survival or death associated with the episode of $P$. aeruginosa sepsis was evaluated as described (10). Clinical characteristics of the patients are described in Table I.

Table I. Characteristics of Patients with IT-2 P. aeruginosa Sepsis

\begin{tabular}{lll}
\hline & Patients & \\
\cline { 2 - 3 } Characteristic & Survivors & Deceased \\
\hline No. & 7 & 6 \\
Mean age (range) & $64(43-82)$ & 60 (23-86) \\
Sex & & \\
$\quad$ Males & 5 & 3 \\
Females & 2 & 3 \\
Neutropenic $\left(<1,000 / \mathrm{mm}^{3}\right)$ & & \\
$\quad$ Yes & 4 & 4 \\
$\quad$ No & 3 & 2 \\
Underlying disease* & & \\
$\quad$ Rapidly fatal & & 1 \\
$\quad$ Ultimately fatal & 1 & 5 \\
Nonfatal & 4 & 0 \\
\hline
\end{tabular}

* Based on classification of McCabe and Jackson (33).
Serologic methods. Serum antibody levels to high molecular weight PS from the IT- 1 through IT-7 strains of $P$. aeruginosa were quantitated by use of a radioactive antigen binding (Farr) test as described $(16,17)$. Opsonophagocytosis assays were also performed as previously described $(17,22)$. Determination of the Ig isotype response to vaccination was evaluated in a radioimmunoprecipitin (RIP) assay as described elsewhere (23).

Statistical methods. Differences in antibody concentrations of preimmunization and postimmunization sera were determined by Student's $t$ test (24) using geometric differences. Opsonophagocytic titers in preimmune and postimmune sera were compared by chi-square analysis. Antibody concentrations in the sera obtained from patient and volunteer groups were compared by a Mann-Whitney U test (24).

\section{Results}

Chemical analyses. The analyses for various biochemical components and monosaccharide constituents in the IT-2 PS vaccine before packaging for human use are shown in Table II. The preparation was principally carbohydrate, with only trace amounts of proteins, nucleic acids, and lipids. The monosaccharides 2-acetamido-2,6-dideoxygalactose ( $N$-acetyl-fucosamine) and glucose were identified with a molar ratio of 2:1.

Structural analysis. The proton $\mathrm{nmr}$ spectrum obtained for the IT-2 high molecular weight PS was identical to those published by two separate groups for the O-specific side chain of the IT-2 $P$. aeruginosa $\operatorname{LPS}(24,25)$. The proton $\mathrm{nmr}$ spectrum contained signals for two $N$-acetyl groups at 2.02 and 2.06 parts per million (ppm), two methyl groups at $1.32 \mathrm{ppm}$, and three anomeric carbons at $4.57,4.70$, and $5.04 \mathrm{ppm}$. The carbon-13 $\mathrm{nmr}$ spectrum was identical to that published by Horton et al. (25) for the IT-2 O-specific side chain, having a doublet signal at 16.3 and $16.4 \mathrm{ppm}$ for the two methyl groups, two doublet signals at 23.3 and $23.2 \mathrm{ppm}$ and 175.1 and $175.4 \mathrm{ppm}$ for acetyl groups, and signals for the anomeric carbons at 100.0 ppm and $103.9 \mathrm{ppm}$. Methylation analysis and Smith degradation analysis were not performed. Since our chemical analysis of the IT-2 high molecular weight PS was identical to that of the IT-2 O-specific side chain, these data show that PS likely has a structure identical to that of the O-specific side chain, which is a repeating unit of: -3$) \mathrm{L}$-fucosamine $(\alpha 1-3)$ D-fucosamine $(\beta 1-2)$ D-glucose $(\beta 1-$.

Molecular weight. The average molecular weight of the IT2 PS vaccine before packaging for human use was determined to be 250,000 on a high performance liquid chromatography

\section{Table II. Biochemical Components and Monosaccharide} Constituents of IT-2 PS Vaccine

Component

\begin{tabular}{|c|c|}
\hline Carbohydrate (total) & 82.2 \\
\hline Lipid & ND \\
\hline Phosphate & ND \\
\hline Nucleic acid & 0 \\
\hline Protein & 0.3 \\
\hline Water & 17.4 \\
\hline Monosaccharide constituents & \\
\hline Glucose & 33 \\
\hline 2-amino-2,6-dideoxygalactose & 67 \\
\hline
\end{tabular}

\footnotetext{
* ND, none detectable
} 
sizing column, when compared to dextran standards. The elution profile was symmetrical and unimodal as measured by absorbance at $206 \mathrm{~nm}$. The $\mathrm{O}$-specific side chain analyzed structurally by Horton et al. (21) had an average molecular weight of 14,000, whereas $\mathrm{O}$ side chains prepared in our laboratory had an average molecular weight of 30,000 (15). These data, coupled with the chemical and structural analyses, indicate that the IT-2 PS is a high molecular weight form of the IT-2 O-specific side chain.

Animal studies. After packaging for human use the IT-2 PS vaccine passed general safety tests in guinea pigs and mice and pyrogenicity tests in rabbits. It took 50,000 times more IT-2 PS than Escherichia coli LPS to gel a limulus lysate $(10 \mu \mathrm{g} / \mathrm{ml} \mathrm{vs}$. $0.2 \mathrm{ng} / \mathrm{ml}$ ).

Toxicity in humans. Three different doses were given to volunteers: five persons received $50 \mu \mathrm{g}$, five persons received $75 \mu \mathrm{g}$, and ten persons received $100 \mu \mathrm{g}$. No local reaction other than tenderness at the injection site was noted by any vaccinate. Tenderness resolved within $24 \mathrm{~h}$. One person given $100 \mu \mathrm{g}$ of vaccine reported a headache upon awakening the day after vaccination. Otherwise, no systemic reactions were noted.

Immune response. Immune sera were obtained from 19 of 20 subjects 2 and 4 wk after immunization. The antibody responses to the vaccine are shown in Table III. All three doses induced significant $(P<0.05)$ increases in binding antibody at 2 and $4 \mathrm{wk}$. Although the higher dose $(100 \mu \mathrm{g})$ elicited a greater geometric mean antibody level, it was not significantly $(P>0.2)$ different from levels achieved by the lower doses. One person given $100 \mu \mathrm{g}$ and one given $75 \mu \mathrm{g}$ failed to increase their antibody level twofold or more, giving an overall response rate of $89 \%$ (17 of 19). No significant $(P>0.5)$ differences were noted in antibody levels achieved at 2 wk vs. 4 wk.

An opsonophagocytosis assay was used to measure antibodymediated killing of $P$. aeruginosa (Table IV). We determined the titer of each serum required to kill $70 \%$ or more of the input inoculum of bacteria. There was a highly significant $(P<0.001)$ increase in opsonic killing titer following immunization. The opsonic titers obtained by immunizing with a single dose of PS were comparable with those seen in humans given five to eight doses of a $P$. aeruginosa LPS vaccine (26).

The isotypes of antibodies specific to IT-2 PS in pre- and

Table III. Antibody Responses to IT-2 PS Vaccine

\begin{tabular}{|c|c|c|c|c|}
\hline \multirow[b]{3}{*}{ Dose } & \multirow[b]{3}{*}{ No. } & \multicolumn{3}{|c|}{$\begin{array}{l}\text { Antibody concentrations: geometric mean } \\
(95 \% \text { confidence interval) }(\mu \mathrm{g} / \mathrm{ml})\end{array}$} \\
\hline & & \multicolumn{3}{|c|}{ Weeks after immunization } \\
\hline & & 0 & 2 & 4 \\
\hline \multicolumn{5}{|l|}{$\mu g$} \\
\hline 50 & 5 & $\begin{array}{l}1.1 \\
(0.3-4.2)\end{array}$ & $\begin{array}{l}6.1^{*} \\
(0.9-39.0)\end{array}$ & $\begin{array}{l}7.4^{*} \\
(1.2-47.4)\end{array}$ \\
\hline 75 & 5 & $\begin{array}{l}1.8 \\
(0.5-6.8)\end{array}$ & $\begin{array}{l}7.3^{*} \\
(1.5-35.0)\end{array}$ & $\begin{array}{l}8.0^{*} \\
(2.0-32.0)\end{array}$ \\
\hline 100 & 9 & $\begin{array}{l}1.1 \\
(0.4-2.9)\end{array}$ & $\begin{array}{l}10.0 \dagger \\
(1.7-60.0)\end{array}$ & $\begin{array}{l}13.8 \dagger \\
(2.2-85.6)\end{array}$ \\
\hline
\end{tabular}

* $P<0.04->0.01$ by a $t$ test of the geometric difference of pre- and postimmunization antibody concentration.

$\dagger P<0.01-<0.0001$ by a $t$ test of the geometric difference of pre- and postimmunization antibody concentration.
Table IV. Opsonophagocytic Killing Titers in Serum Obtained from Vaccinates Given IT-2 PS

\begin{tabular}{lccr}
\hline Serum & Titer & & \\
\hline & $\leq 4$ & $8-32$ & $\geq 64$ \\
Preimmunization & $17^{*}$ & 2 & 0 \\
Postimmunization & & & 12 \\
2 wk $\dagger$ & 2 & 5 & 13 \\
4 wk $\dagger$ & 2 & 4 & \\
\hline
\end{tabular}

* Represents number of vaccinates with this titer.

$\dagger P<0.001$ vs. preimmunization titers by chi-square analysis. Cells containing titers of 8-32 and $\geq 64$ collapsed together for statistical analysis.

postimmunization sera were determined by an RIP test (23) (Table V). The percentages of IgG, IgM, and IgA specific for IT2 PS in preimmune serum were roughly equal, with a slight preponderance of IgA. In postimmunization sera, the proportion of specific antibody that was IgG rose, the proportion of specific IgM fell, and the proportion of specific IgA increased slightly. The total concentration of specific antibody was higher for each isotype after immunization, and the increases were most pronounced in the $\operatorname{IgG}$ and $\operatorname{IgA}$ fractions.

Vaccinates given IT-2 PS did not develop measureable antibody responses to heterologous PS isolated from the IT-1 and 3-7 strains of $P$. aeruginosa. Furthermore, there were no detectable hemagglutinating antibody responses to ABO blood group antigens in either vaccinate sera or high titered immune rabbit sera raised to IT-2 $P$. aeruginosa bacterial cells.

Comparison of IT-2 PS-specific antibody levels in patients and vaccinates. Pollock and Young $(10)$ have shown that elevated levels of hemagglutinating antibody to LPS in acute-phase serum is associated with survival of $P$. aeruginosa bacteremia. We examined the concentration of antibody to IT-2 PS in the acute-

Table V. Immunoglobulin Isotype Responses of the Vaccinates to IT-2 PS

\begin{tabular}{|c|c|c|c|}
\hline \multirow[b]{2}{*}{ Sera from week } & \multicolumn{3}{|c|}{$\begin{array}{l}\text { Percent* }( \pm \text { SD) and concentration } \dagger( \pm \text { SD) of } \\
\text { IT-2 PS-specific antibody in vaccinate sera }\end{array}$} \\
\hline & IgM & IgG & IgA \\
\hline \multicolumn{4}{|l|}{0 wk } \\
\hline Percent & $32 \pm 17$ & $27 \pm 16$ & $39 \pm 11$ \\
\hline Concentration $(\mu g / m l)$ & $0.4 \pm 0.2$ & $0.4 \pm 0.2$ & $0.5 \pm 0.1$ \\
\hline \multicolumn{4}{|l|}{$2 \mathrm{wk}$} \\
\hline Percent & $14 \pm 11$ & $38 \pm 17$ & $45 \pm 14$ \\
\hline Concentration $(\mu g / m l)$ & $1.2 \pm 0.9$ & $3.2 \pm 1.4$ & $3.8 \pm 1.2$ \\
\hline \multicolumn{4}{|l|}{$4 \mathrm{wk}$} \\
\hline Percent & $14 \pm 12$ & $46 \pm 18$ & $42 \pm 16$ \\
\hline Concentration $(\mu g / m l)$ & $1.5 \pm 1.3$ & $4.9 \pm 1.9$ & $4.4 \pm 1.7$ \\
\hline
\end{tabular}

* Percent of each isotype determined by a RIP test (22), wherein ${ }^{14} \mathrm{C}$ labeled PS was added to vaccinate sera, incubated at $37^{\circ} \mathrm{C}$ for $2 \mathrm{~h}$, and the resultant antigen-antibody complexes precipitated with goat antihuman IgM, IgG, or IgA at $4^{\circ} \mathrm{C}$ for $18 \mathrm{~h}$.

$\dagger$ Calculated using a radioactive antigen binding assay as described $(16,17)$. 
Table VI. Antibody Concentrations Specific

for IT-2 PS in Bacteremic Patients

\begin{tabular}{llc}
\hline $\begin{array}{l}\text { Patient } \\
\text { status }\end{array}$ & No. & $\begin{array}{l}\text { Antibody concentration: geometric mean } \\
(95 \% \text { confidence interval })(\mu g / m l)\end{array}$ \\
\hline $\begin{array}{l}\text { Survived } \\
\text { Died }\end{array}$ & 7 & $12.8(1.6-102.4)$ \\
& 6 & $2.0(0.2-22.4)^{*}$ \\
\hline
\end{tabular}

* $P=0.05$ by a Mann-Whitney $U$ test compared with survivors.

phase sera of 13 patients with IT-2 $P$. aeruginosa sepsis. There was a significant difference $(P=0.05)$ in antibody levels between patients who survived and those who died within $72 \mathrm{~h}$ of a positive blood culture for $P$. aeruginosa (Table VI). Antibody levels in survivors did not differ significantly from those of vaccinates 2 and 4 wk after immunization $(P=0.39$ and 0.56 , respectively). Antibody levels in vaccinates 2 and 4 wk after immunization were significantly greater $(P=0.03$ and 0.02 , respectively) than levels found in patients who died. Preimmunization antibody levels in vaccinates were comparable with those of patients who died $(P=0.97)$. Vaccination appeared to elicit antibody levels that are associated with, though not necessarily responsible for, survival from $P$. aeruginosa sepsis.

\section{Discussion}

The results reported here provide evidence that IT-2 PS has potential to function as a vaccine against some strains of $P$. aeruginosa. We showed that IT-2 PS is chemically and structurally identical to the $\mathrm{O}$-specific side chain, which expresses the serotype antigens in $P$. aeruginosa. The average molecular weight of IT-2 PS is nearly 20 times that of the O-specific side chain. This large size, the lack of animal toxicity, and the lack of chemical components thought responsible for toxicity are important features of polysaccharide vaccines. The safety for human administration of the preparation of IT-2 PS described here was demonstrated by the minimal adverse effects experienced by vaccinates. The immunogenicity of IT-2 PS was evidenced by the antibody response of vaccinates. We also showed that antibody levels to IT-2 PS were higher in patients who survived IT-2 P. aeruginosa bacteremia than those of patients who died. It is of potential clinical significance that these antibody concentrations were achieved within 2 wk of vaccinating healthy humans with a single dose of IT-2 PS.

We believe that the development of an immunogenic form of the O-specific side chain that can be administered without significant toxicity constitutes a major step towards development of a $P$. aeruginosa vaccine. Numerous animal studies $(9,27)$ have demonstrated that antibody specific for serotype antigens such as high molecular weight PS are protective against $P$. aeruginosa infection. Previous attempts to vaccinate humans with $P$. aeruginosa LPS were hampered by the toxicity of this vaccine (12). High molecular weight PS apparently is without such toxicity, yet retains the desired immunogenic and serologic properties.

Animal studies have indicated that antibody to the $P$. aeruginosa serotype antigen is sufficient, by itself, to afford complete protection against bacterial challenge. Mice given a $2.5 \mathrm{~cm}^{2}$ thermal injury are protected against challenge with $10^{7}-10^{8}$ bacteria following active and passive immunization with PS or an- tisera to PS $(14,28)$. As little as $1 \mu \mathrm{g}$ of passively transferred antibody was able to confer protection. Sawada et al. (29) recently showed in a murine model that $0.05-2.5 \mu \mathrm{g} / \mathrm{mouse}$ of LPS-specific monoclonal antibodies prevented systemic spread of $\boldsymbol{P}$. aeruginosa after infection of thermal injuries. Despite the variety of virulence factors produced by $P$. aeruginosa, systemic infection can apparently be prevented by type-specific antibody alone.

An important consequence of our study has been the determination of type-specific antibody levels associated with survival of $P$. aeruginosa sepsis. Numerous commercial preparations of intravenous IgG are currently being evaluated and marketed, with indications that they may be useful in treating infections with $P$. aeruginosa $(30,31)$. Our finding that survivors had an average type-specific antibody level of $12.8 \mu \mathrm{g} / \mathrm{ml}$ suggests a concentration of antibody that may need to be achieved in order to assure therapeutic efficacy. Only one of eight patients with antibody levels $\geq 3.0 \mu \mathrm{g} / \mathrm{ml}$ died, while five of six patients with lower concentrations died. Thus $3.0 \mu \mathrm{g} / \mathrm{ml}$ may be a lower limit of type-specific antibody concentration that possibly gives immunologic protection against $P$. aeruginosa infections.

Our finding that an IT-2 PS vaccine elicits antibody levels that are comparable with those in bacteremic patients who survive IT-2 $P$. aeruginosa sepsis suggests that the vaccine may, in fact, offer protection in humans. Interpretation of these data is confounded by the fact that antibody levels in acute-phase sera may be lowered because of antigen release during infection. In addition, numerous host factors contribute to the outcome of $P$. aeruginosa sepsis, so that elevated antibody titers cannot conclusively be said to have been protective. We did not perform a multiple regression analysis on clinical factors associated with the infections, so variables other than antibody titer may also have contributed to this outcome. In a similar study, however, Pollock and Young (10) did not identify any clinical factors contributing to the outcome of bacteremia other than antibody levels to $P$. aeruginosa antigens. In our study, one patient with low antibody levels to IT-2 PS survived, whereas the patient with the highest level of antibody died. Clearly, factors other than antibody to PS can be involved in the clinical outcome. Moreover, we did not analyze serum antibody levels to exotoxin A, as did Pollock and Young (10) and Cross et al. (32). These studies did show a correlation between survival of $P$. aeruginosa sepsis and elevated levels of antibody to exotoxin A. The latter study, however, did not control for antibody to serotype antigens, which the former study showed to be correlated with antibody levels to exotoxin A $(r=0.33, P=0.06)(10)$. In conclusion, our demonstration of comparable antibody levels in survivors of bacteremia and vaccinates is encouraging but by no means confirms that vaccination with IT-2 PS will be protective.

\section{Acknowledgment}

This work was supported by grant No. AI22535 from the National Institute of Allergy and Infectious Diseases.

\section{References}

1. Reynolds, H. Y., A. S. Levine, R. E. Wood, C. H. Zierdt, and D. C. Dale. 1975. Pseudomonas aeruginosa infections: persisting problems and current research to find new therapies. Ann. Intern. Med. 82: 819-827.

2. di Sant' Agnese, P. A., and P. B. Davis. 1979. Cystic fibrosis in adults: 75 cases and a review of 232 cases in the literature. Am. J. Med. 66:121-132. 
3. Schimpff, S. C., W. H. Greene, V. M. Young, and P. H. Wiernik. 1974. Significance of Pseudomonas aeruginosa in the patient with leukemia or lymphoma. J. Infect. Dis. 130(Suppl):524-531.

4. Pennington, J. E., H. Y. Reynolds, and P. P. Carbone. 1973. Pseudomonas pneumonia: a retrospective study of 36 cases. Am. J. Med. 55: $155-160$.

5. Gilbert, D. N. 1985. An evaluation of anti-pseudomonal antimicrobic agents. In Pseudomonas aeruginosa: New Therapeutic Approaches from Basic Research. D. P. Speert and R. E. W. Hancock, editors. S. Karger, Basel, Switzerland. 111-133.

6. Bryan, L. E. 1979. Resistance to antimicrobial agents: the general nature of the problem and the bases of resistance. In Pseudomonas aeruginosa: Clinical Manifestations of Infection and Current Therapy. R. G. Doggett, editor. Academic Press. New York. 219-271.

7. Crowder, J. G., M. W. Fisher, and A. White. 1972. Type specific immunity in Pseudomonas diseases. J. Lab. Clin. Med. 79:47-54.

8. Young, L. S., B. H. Yu, and D. Armstrong. 1970. Agar gel precipitating antibody in Pseudomonas aeruginosa infections. Infect. Immun. 2:495-503.

9. Hanessian, S., W. Regan, D. Watson, and T. H. Haskell. 1971. Isolation and characterization of the antigenic components of a new heptavalent Pseudomonas vaccine. Nature New Biol. (Lond.). 229:209210.

10. Pollack, M., and L. S. Young. 1979. Protective activity of antibodies to exotoxin A and lipopolysaccharide at the onset of Pseudomonas aeruginosa septicemia in man. J. Clin. Invest. 63:276-286.

11. Pollack, M., A. I. Huang, R. K. Prescott, L. S. Young, K. W. Hunter, D. F. Cruess, and C. M. Tsai. 1983. Enhanced survival in Pseudomonas aeruginosa septicemia associated with high levels of circulating antibody to Escherichia coli endotoxin core. J. Clin. Invest. 72:18741881.

12. Pennington, J. E. 1974. Preliminary investigations of Pseudomonas aeruginosa vaccine in patients with leukemia and cystic fibrosis. J. Infect. Dis. 130(Suppl):S159-S162.

13. Jones, R. J., E. A. Roe, and J. L. Gupta. 1979. Controlled trial of a polyvalent pseudomonas vaccine in burns. Lancet. ii:977-983.

14. Pier, G. B., M. Pollack, and M. Cohen. 1984. Immunochemical characterization of high-molecular weight polysaccharide from Fisher Immunotype 3 Pseudomonas aeruginosa. Infect. Immun. 45:309-319.

15. Pier, G. B., H. F. Sideberry, and J. C. Sadoff. 1981. High-molecular weight polysaccharide antigen from Pseudomonas aeruginosa immunotype 2. Infect. Immun. 34:461-468.

16. Pier, G. B. 1982. Cross-protection by Pseudomonas aeruginosa polysaccharides. Infect. Immun. 38:1117-1122.

17. Pier, G. B. 1982. Safety and immunogenicity of high molecular weight polysaccharide vaccine from immunotype 1 Pseudomonas aeruginosa. J. Clin. Invest. 69:303-308.

18. Pier, G. B., M. Cohen, and H. Jennings. 1983. Further purification and characterization of high-molecular weight polysaccharide from Pseudomonas aeruginosa. Infect. Immun. 42:936-941.

19. Brokopp, C. D., and J. J. Farmer. 1979. Typing methods for
Pseudomonas aeruginosa. In Pseudomonas aeruginosa: Clinical Manifestations of Infection and Current Therapy. Academic Press. New York 90-134.

20. Keleti, G., and W. H. Lederer. 1974. Handbook of Micromethods for the Biological Sciences. Van Nostrand Reinhold Co., New York. 72.

21. Horton, D., D. A. Riley, S. Samreth, and M. G. Schweitzer. 1983. Lipopolysaccharide antigens of Pseudomonas aeruginosa. In Bacterial Lipopolysaccharides: Structure, Synthesis, and Biological Activities. L. Anderson and F. M. Unger, editors. American Chemical Society. New York. 21-47.

22. Peterson, P. K., Y. Kim, D. Schmelang, M. Lindemann, J. Verhoff, and P. G. Quie. 1978. Complement mediated phagocytosis of Pseudomonas aeruginosa. J. Lab. Clin. Med. 92:883-894.

23. Käyhty, H., H. Jousimies-Somer, H. Peltola, and H. Mäkelä. 1981. Antibody response to capsular polysaccharides of groups $A$ and $C$ Neisseria meningitidis and Haemophilus influenzae type b bacteremic disease. J. Infect. Dis. 143:32-41.

24. Finney, D. J. 1978. Statistical Methods in Biological Assay. Macmillan Publishing Co., Inc., New York. Third ed. 394-410.

25. Dimitriev, B. A., Y. A. Knirel, N. A. Kocharova, N. K. Kochetkov, E. S. Stanislavsky, and G. M. Mashilova. 1980. Somatic antigens of Pseudomonas aeruginosa. The structure of the polysaccharide chain of Ps. aeruginosa O-serogroup 7 (Lanyi) lipopolysaccharide. Eur. J. Biochem. 106:643-651.

26. Young, L. S. 1972. Human immunity to Pseudomonas aeruginosa. II. Relationship between heat stable opsonins and type-specific lipopolysaccharides. J. Infect. Dis. 126:277-287.

27. Alms, T. H., and J. A. Bass. 1967. Immunization against Pseudomonas aeruginosa. 1 . Induction of protection by an alcohol precipitated fraction from the slime layer. J. Infect. Dis. 117:249-256.

28. Pollack, M., G. B. Pier, and R. K. Prescott. 1984. Immunization with Pseudomonas aeruginosa high-molecular-weight polysaccharides prevents death from Pseudomonas burn infections in mice. Infect. Immun. 43:759-760.

29. Sawada, S., M. Suzuki, T. Kawamura, S. Fujinaga, Y. Masuho, and K. Tomibe. 1984. Protection against infection with Pseudomonas aeruginosa by passive transfer of monoclonal antibodies to lipopolysaccharides and outer membrane proteins. J. Infect. Dis. 150:570-576.

30. Pollock, M. 1983. Antibody activity against Pseudomonas aeruginosa in immune globulins prepared for intravenous use in humans. $J$. Infect. Dis. 147:1090-1098.

31. Collins, M. S., and J. H. Dorsey. 1984. Comparative anti-Pseudomonas aeruginosa activity of chemically modified and native immunoglobulin $G$ (human) and potentiation of antibiotic protection against Pseudomonas aeruginosa and group B Streptococcus in vivo. Am. J. Med. 76:155-160.

32. Cross, A. S., J. C. Sadoff, B. H. Iglewski, and P. A. Sokol. 1980 Evidence for the role of toxin $A$ in the pathogenesis of infection with Pseudomonas aeruginosa in humans. J. Infect. Dis. 142:538-546.

33. McCabe, W. R., and G. G. Jackson. 1962. Gram-negative bacteremia. I. Etiology and ecology. Arch. Intern. Med. 110:847-855. 